\section{Experiencia de caries dental en trabajadores universitarios: su asociación con variables sociodemográficas}

\section{Caries experience in university workers: its association with sociodemographic data}

\begin{abstract}
Resumen
Objetivo. Evaluar la experiencia de caries en trabajadores universitarios y su asociación con variables sociodemográficos. Métodos. Estudio transversal, observacional y analítico con un grupo de trabajadores de la Universidad de Guadalajara, México. Se recopilaron datos de las variables sociodemográficas mediante un cuestionario y a través del examen clínico el índice CPOD. Se realizaron pruebas estadísticas de Chi-cuadrado, t-Student, ANOVA de una vía y post-hoc con significancia de 0,05. Resultados. Doscientos noventa participantes accedieron a la exploración oral, con un promedio de edad de 36,2 \pm 0,6, 55,9\% fueron mujeres. El CPOD de la muestra evaluada fue de 8,3 $\pm 5,3$. El análisis de Chi-cuadrado en la evaluación etaria y los niveles de CPOD mostraron significancia estadística $(p=0,0001)$. De forma similar, en las pruebas de ANOVA de una vía en el grupo de hombres $(p=0,0001)$ y en los que residen en Tonalá $(p=0,022)$, el componente cariados del índice se vio significativamente aumentado. Se observó también, un índice aumentado de dientes obturados en los participantes con nivel máximo de posgrado $(p=0,001)$. El grupo de fumadores mostró significancia estadística $(p=0,034)$ en el índice de caries. Conclusiones. El índice CPOD poblacional se encontró en nivel bajo, estando el grupo de mayor edad significativamente por encima de los dos grupos de menor edad. Los niveles del componente cariados fueron más bajos en los habitantes de las regiones foráneas del estado y los que tenían escolaridad de posgrado $(p=0,022$ y $p=0,02$ respectivamente).
\end{abstract}

Palabras clave: Caries dental; Trabajadores; Adultos (fuente: DeCS BIREME).

\section{Artículo Original}

Claudia Vázquez-Bojórquez ${ }^{1, a}$, César Octavio De Loera-Rodríguez ${ }^{2, b}$, Sandra López-Verdín ${ }^{3, c}$, Luis Miguel Miramontes-Díaz 1,a, Israel Guardado-Luévanos s,a.

${ }^{1}$ Universidad Autónoma Metropolitana, Ciudad de México, México

${ }^{2}$ Universidad de Guadalajara, Centro Universitario de Ciencias de la Salud, Departamento de Fisiología, Jalisco, México.

${ }^{3}$ Universidad de Guadalajara, Centro Universitario de Ciencias de la Salud, Instituto de Investigación en Odontología, Jalisco, México.

${ }^{4}$ Universidad de Guadalajara, Centro Universitario de Ciencias de la Salud, Jalisco, México.

${ }^{a}$ Licenciado en Cirujano Dentista.

${ }^{\circ}$ Doctor en Ciencias Biomédicas.

${ }^{\circ}$ Doctora Ciencias en Biología Molecular en Medicina.

\section{Correspondencia:}

Sandra López-Verdín: sandra.Iverdin@academicos.udg.mx Sierra Mojada 950, Edificio "C", Primera Planta, Col. Independencia, C.P. 44340, Guadalajara, Jalisco, México. ORCID: 0000-0002-8397-1720

\section{Coautores:}

Claudia Vázquez-Bojórquez: clauboj@gmail.com ORCID: 0000-0002-4097-8973

Cesar Octavio DeLoera-Rodríguez: cesar.deloera@red. udg.mx

Luis Miguel Miramontes Díaz: luismidi@gmail.com ORCID: 0000-0002-5238-5112

Israel Guardado-Luévanos: iguardadol@hotmail.com ORCID: 0000-0002-9847-5041

\section{Editora:}

Marieta Petkova-Gueorguieva

Universidad Nacional Mayor de San Marcos, Perú.

Conflicto de intereses: Los autores declaran no tener conflicto de interés.

Fuente de financiamiento: autofinanciado

Recibido: 06/11/19

Aceptado: $28 / 03 / 20$

Publicado: 09/05/20 
Women represented 55.9\%. The DMFT of the evaluated sample was $8.3 \pm 5.3$. The $\chi 2$ analysis of the evaluated age ratio, showed statistical significance $(\mathrm{p}=0.0001)$. Similarly, the decay component of the index was increased in the one-way ANOVA test of the male group $(p=0,0001)$ and of those who reside in Tonala $(p=0.022)$. Additionally, an increased number of filled teeth was found in participants with postgraduate education $(\mathrm{p}=0.001)$. The smokers group showed statistical significance $(\mathrm{p}=0.034)$ in caries index. Conclusions. The broad DMFT index was found at a low level, however, the older age group was significantly different, being at the high level, well above the two younger age groups. The caries component of the DMFT index was significantly lower in the out of the region residents and those who had postgraduate degree $(p=0.022$ and $p=0.02$ respectively).

Keywords: Dental caries; Workers; Adults (source: MeSH NLM).

\section{Introducción}

La caries dental es una enfermedad compleja, multifactorial que resulta de la interacción de las bacterias acidófilas del biofilm que al exponerse a los carbohidratos dietéticos producen ácidos orgánicos débiles que provocarán una desmineralización y cavitación a través de la capa del esmalte. Cuando la enfermedad no es tratada y se permite su progresión, ésta puede dañar capas más profundas a través de la dentina y llegar a la pulpa dental, derivando en pérdida dental en los casos más avanzados ${ }^{1}$.

La caries dental continúa siendo la enfermedad más prevalente a nivel mundial, además puede ocasionar dolor, pérdida de la función y otras dificultades derivadas de falta de atención, lo que comprometen a la salud bucal y al bienestar de la población, interviniendo directamente en la calidad de vida ${ }^{2,3}$. Aunque la información sobre caries en adultos no es abundante como sobre la caries en edad infantil, se ha reportado que tiene una alta prevalencia en la población, hasta del 99\% en adultos mexicanos ${ }^{4}$, por lo que se considera de suma importancia dirigir estudios en este sector poblacional.

El estudio de las condiciones bucales como caries dental, restauraciones dentales permanentes y pérdida de dientes a través del índice de dientes cariados, perdidos y obturados (CPOD), provee un modelo ideal para investigar el impacto de la enfermedad en la población ${ }^{5}$. Este índice ofrece un panorama de la enfermedad presente y pasada en dentición permanente, además de brindar un panorama etiológico que comprende un conjunto de factores sociales, biológicos y de comportamiento, debido a que se ha reportado con anterioridad que la situación socioeconómica y sociodemográfica de los individuos afecta su salud bucal y general ${ }^{6}$.

La prevalencia de caries dental y sus consecuencias han sido asociadas a múltiples factores tales como etnicidad, ingresos, nivel educativo, nivel socioeconómico, zona de residencia como predictoras de la ocurrencia de la enfermedad ${ }^{7}$. Incluso, existen estudios que sugieren que la cantidad de caries aumenta, particularmente en los grupos de adultos, con hábitos de tabaco y alcohol, esto posiblemente relacionado a la deficiente salud bucal en estos ${ }^{8-10}$.
El estudio se enfocó en una población que incluye adultos jóvenes y adultos mayores, lo que podría contribuir a la obtención de datos de la experiencia de caries en la población adulta en general. Los trabajadores evaluados que laboran en la Universidad de Guadalajara cuentan con un ingreso económico estable y prestaciones laborales otorgadas por la ley, las cuales incluyen un seguro social en salud, lo que brindaría un panorama favorable para padecer niveles bajos de caries dental.

El objetivo del estudio fue evaluar la experiencia de caries en trabajadores universitarios y asociarla con los variables sociodemográficos de este grupo poblacional.

\section{Métodos}

Se diseñó un estudio transversal, observacional y analítico con un grupo, el cual fue evaluado y aceptado por el Comité de ética del Centro Universitario de Ciencias de la Salud (dcto n09/2019). Se llevó a cabo en los diferentes Centros Universitarios de la Universidad de Guadalajara ubicados dentro de la zona metropolitana del estado de Jalisco, México.

Se realizó un muestreo por conveniencia donde se incluyeron a los trabajadores adscritos a la Universidad de Guadalajara que contaran con código de trabajador y nombramiento vigente durante el periodo de enero de 2017 y marzo del 2018. Los participantes fueron invitados al estudio a través de un consentimiento informado escrito, en el cual firmaron y aceptaron su inclusión. Éste se apegó a los principios de la Declaración de Helsinki para estudios no experimentales en humanos ${ }^{11}$.

Los encuestados proporcionaron sus datos sociodemográficos (sexo, residencia, escolaridad) y de hábitos de tabaco y alcohol. Posteriormente el examen clínico fue realizado con luz fría indirecta. La exploración oral fue hecha y registrada por cuadrantes. Los trabajadores que no accedieron a la exploración oral no fueron incluidos en el estudio.

La exploración oral fue realizada por ocho odontólogos examinadores, estandarizados previamente utilizando herramientas fotográficas y simulando el examen clínico en sesiones previas, para obtener el índice CPOD sugerido por la Organización Mundial de la Salud (OMS) ${ }^{12}$ con un valor de coeficiente kappa inter-evaluador de 0,53 y 0,60 intra-evaluador ${ }^{13}$. 
El índice fue evaluado por componentes de acuerdo con los niveles de caries, dientes perdidos y obturaciones, las cuales fueron sumadas posteriormente para obtener el índice CPOD. La escala para evaluar el nivel del Índice CPOD fue propuesta por la OMS en 2013, donde se establece como estándar al grupo etario de adultos (3544 años) y se indica que el nivel muy bajo se encuentra por debajo de 5 , se considera bajo cuando se encuentra entre 5,0-8,9; moderado en 9,0-13,9 y alto cuando es mayor a $13,9^{8}$.

Para el análisis de los datos se crearon tres grupos etarios, el primer grupo corresponde a 19-34 años, el segundo incluye a los sujetos entre 35-44 ańos, el tercero corresponde a los participantes entre 45-64 años. Es importante mencionar que la severidad de caries en adultos de la OMS refiere particularmente al grupo etario de 35-44 años. Debido a falta de estratificación por la OMS de los adultos en distintos grupos etarios como los que se utilizaron en este estudio, y con fines de analizar los niveles de caries en adultos, el grupo de 35 a 44 años será utilizado como el referente de la escala de severidad. Los datos sociodemográficos evaluados fueron la zona de residencia de los participantes y la escolaridad.

Los datos fueron registrados y procesados en el software SPSS Statistics v.20.0 IBM en el cual fueron analizados promedios, frecuencias, prevalencias, índice CPOD. Se realizaron análisis de Chi-cuadrado y t-Student para determinar la diferencia en el índice CPOD entre los grupos evaluados por sexo, edad, residencia y escolaridad. La prueba ANOVA de una vía se utilizó para determinar si las diferencias entre grupos eran significativas para los datos sociodemográficos de zona de residencia y escolaridad para posteriormente analizar la interacción entre los grupos sociodemográficos con un análisis post-hoc (T3 de Dunnett y Bonferrioni, respectivamente). Se estableció el nivel de significancia de 0,05.

\section{Resultados}

De los 630 participantes, 290 fueron los que accedieron a la exploración oral.

El promedio de edad del total de los trabajadores que accedieron a la exploración oral fue de 36,2 $\pm 0,6$. El $55,9 \%(n=162)$ fueron mujeres, mientras que el $44,1 \%$ $(\mathrm{n}=128)$ fueron hombres.

La mayoría de los participantes $(65,1 \%)$ reside en Guadalajara, y el nivel educativo con mayor frecuencia fue Licenciatura $(55,5 \%)$.

El CPOD de la muestra evaluada fue de $8,3 \pm 5,3$ (IC $95 \% 3,6-4,5)$ (Tabla 1). La mayor parte de la población se puede catalogar en un nivel bajo y moderado (Tabla 2).

Tabla 1. Índice CPOD de acuerdo con los datos sociodemográificos

\begin{tabular}{|c|c|c|c|c|c|c|c|c|c|}
\hline Grupos de Edad & $\mathbf{n}$ & $\mathrm{C}$ & IC $95 \%$ & $\mathbf{P}$ & IC $95 \%$ & $\mathrm{O}$ & IC $95 \%$ & CPOD & IC $95 \%$ \\
\hline 19-34 años & 154 & $4,2 \pm 0,3$ & $3,6-4,9$ & $0,7 \pm 0,1$ & $0,4-0,9$ & $2,0 \pm 0,2$ & $1,5-2,5$ & $6,9 \pm 0,4$ & $6,1-7,8$ \\
\hline 35-44 años & 67 & $3,6 \pm 0,4$ & $2,6-4,4$ & $1,1 \pm 0,2$ & $0,6-1,5$ & $3,4 \pm 0,4$ & $2,5-4,4$ & $8,1 \pm 0,5$ & $6,9-9,3$ \\
\hline 45-64 añost & 69 & $4,3 \pm 0,4$ & $3,4-5,3$ & $1,9 \pm 0,3$ & $1,2-2,5$ & $5,4 \pm 0,6$ & $3,8-6,4$ & $11,5 \pm 0,6$ & $10,1-12,7$ \\
\hline Total & 290 & $4,0 \pm 0,2$ & $3,6-4,5$ & $1,0 \pm 0,1$ & $0,8-1,3$ & $3,1 \pm 0,2$ & $2,6-3,6$ & $8,3 \pm 5,3$ & $7,6-8,9$ \\
\hline Valor de $p$ & & $0,390^{\mathrm{a}}$ & & $0,0001^{a, b}$ & & $0,0001^{a, b^{*}}$ & & $0,0001^{a, b}$ & \\
\hline \multicolumn{10}{|l|}{ Sexo } \\
\hline Hombres & 128 & $5,0 \pm 0,3$ & $4,3-5,7$ & $0,9 \pm 0,1$ & $0,5-1,1$ & $2,7 \pm 0,4$ & $1,9-3,3$ & $8,6 \pm 0,5$ & $7,5-9,5$ \\
\hline Mujeres & 162 & $3,4 \pm 0,3$ & $2,7-3,9$ & $1,2 \pm 0,1$ & $0,9-1,5$ & $3,5 \pm 0,3$ & $2,8-4,1$ & $8,1 \pm 0,4$ & $7,2-8,9$ \\
\hline Valor de $p$ & & $0,0001^{*^{*}}$ & & $0,092^{c}$ & & $0,093^{c}$ & & $0,440^{c}$ & \\
\hline \multicolumn{10}{|l|}{ Residencia } \\
\hline Guadalajara & 194 & $4,8 \pm 0,3$ & $3,5-4,5$ & $1,1 \pm 0,1$ & $0,8-1,4$ & $3,1 \pm 0,3$ & $2,5-3,8$ & $8,3 \pm 0,4$ & $7,5-9,1$ \\
\hline Zapopan & 44 & $4,3 \pm 0,6$ & $3,0-5,7$ & $1,3 \pm 0,3$ & $0,6-1,9$ & $2,9 \pm 0,6$ & $1,7-4,2$ & $8,6 \pm 0,9$ & $9,6-10,4$ \\
\hline Tlajomulco & 7 & $6,3 \pm 1,7$ & $1,5-10,7$ & $0,1 \pm 0,1$ & $0,2-0,6$ & $0,8 \pm 0,5$ & $0,5-2,2$ & $7,1 \pm 1,8$ & $2,2-12,0$ \\
\hline Tlaquepaque & 11 & $5,2 \pm 1,9$ & $1,2-9,1$ & $0,5 \pm 0,3$ & $0,2-1,2$ & $2,5 \pm 1,5$ & $0,6-5,6$ & $8,2 \pm 1,9$ & $4,1-12,2$ \\
\hline Tonalá & 11 & $6,8 \pm 1,2$ & $4,2-10,0$ & $1,1 \pm 0,5$ & $1,0-2,5$ & $1,7 \pm 0,6$ & $0,2-3,3$ & $9,6 \pm 1,7$ & $6,1-14,1$ \\
\hline $\mathrm{RFE}^{1}+$ & 23 & $3,8 \pm 0,7$ & $0,9-3,4$ & $0,9 \pm 0,2$ & $0,3-0,9$ & $3,2 \pm 1,0$ & $2,2-6,1$ & $7,9 \pm 1,0$ & $4,9-8,8$ \\
\hline Valor de $p$ & & $0,022^{a, b^{*}}$ & & $0,367^{a}$ & & $0,420^{a}$ & & $0,767^{a}$ & \\
\hline \multicolumn{10}{|l|}{ Escolaridad } \\
\hline E. Básica & 16 & $5,1 \pm 1,0$ & $1,1-13,8$ & $1,0 \pm 0,7$ & $0,2-2,3$ & $2,6 \pm 0,8$ & $1,1-6,4$ & $9,2 \pm 1,5$ & $7,1-28,5$ \\
\hline Preparatoria & 46 & $4,3 \pm 0,5$ & $3,1-5,5$ & $1,5 \pm 0,3$ & $0,8-2,1$ & $1,8 \pm 0,3$ & $0,9-2,9$ & $7,6 \pm 0,7$ & $6,2-9,2$ \\
\hline Licenciatura & 164 & $4,3 \pm 0,3$ & $3,7-5,0$ & $1,0 \pm 0,1$ & $0,7-1,3$ & $2,9 \pm 0,3$ & $2,2-3,3$ & $8,3 \pm 0,4$ & $7,3-9,2$ \\
\hline Posgrado† & 64 & $2,9 \pm 0,6$ & $1,4-4.9$ & $0,9 \pm 0,3$ & $0,3-1,5$ & $4,9 \pm 0,7$ & $2,6-8,8$ & $8,7 \pm 0,5$ & $6,9-12,1$ \\
\hline Valor de $p$ & & $0,020^{\mathrm{a}, \mathrm{d}^{*}}$ & & $0,804^{a}$ & & $0,001^{a, d^{*}}$ & & $0,682^{\mathrm{a}}$ & \\
\hline
\end{tabular}

C; Cariados, P; Perdidos, O; Obturados, CPOD; Índice de dientes cariados, perdidos y obturados. Se realizó análisis de One way ANOVA para la comparación entre grupos, b Post hoc test T3 Dunnet c Se realizó análisis T-student d Post-hoc Test Bonferroni *Significancia estadística $p=<0,05$ +Grupo significativo en el análisis Post-Hoc. ${ }^{1}$ RFE: Regiones Foráneas del Estado. 
Se realizaron pruebas de Chi-cuadrado en relación con los datos sociodemográficos, donde se distinguió que el CPOD de los grupos de edad se mostraba significativamente diferente entre sí $(p=0,0001, \mathrm{Chi}-2)$. Por otro lado, durante este análisis los datos de sexo $(p=0,515$, Chi-2), escolaridad ( $p=0,917$, Chi-2,) y residencia $(p=0,904, \mathrm{Chi}-2)$ no mostraron diferencias significativas (Tabla 2).

Es importante resaltar que, el análisis del Índice por componentes mostró que el grupo de mayor edad reporta mayor índice CPOD debido a la mayor cantidad de dientes perdidos y obturados (Tabla 1).

El análisis de Chi-cuadrado no fue significativo cuando se evaluó el índice en base a la escala de severidad de la OMS y el sexo, sin embargo, por componentes, el índice de dientes cariados se ve significativamente aumentado en el sexo masculino en comparación con el femenino ( 5,0 vs 3,$4 ; p=0,0001)$, y aunque esto genera una diferencia en el índice CPOD, no es de manera significativa, debido a que las mujeres presentan mayor índice de obturaciones y de dientes perdidos (Tabla 1).
El análisis del índice CPOD, en relación con la residencia mostró diferencias significativas donde el mayor índice lo mostraron los que residen en Tonalá (prueba de ANOVA de una vía y test post-hoc de T3 de Dunnet), mientras que el índice de los que residen en regiones foráneas del estado fue significativamente menor en comparación con los demás grupos (Tabla 1).

Los individuos que contaron con un nivel máximo de posgrado presentaron significativamente menor cantidad de dientes cariados $(p=0,02)$ y mayor cantidad de obturados $(p=0,001)$, aunque el valor global de CPOD no se muestra diferente de manera significativa con los otros los niveles de escolaridad (prueba ANOVA de una vía y test post-hoc de Bonferrioni) (Tabla 1).

Se analizó el índice CPOD acumulado y de los componentes de este en relación con los hábitos de consumo de tabaco y alcohol, donde se encontró que el índice de dientes cariados en el grupo de fumadores es significativamente mayor en comparación con los no fumadores $(\mathrm{p}=0,034)$. No se observaron diferencias significativas

Tabla 2. Distribución de los niveles (OMS) del índice CPOD de acuerdo con los datos sociodemográficos

\begin{tabular}{|c|c|c|c|c|c|c|c|c|c|c|c|}
\hline & \multicolumn{2}{|c|}{ Muy bajo } & \multicolumn{2}{|c|}{ Bajo } & \multicolumn{2}{|c|}{ Moderado } & \multicolumn{2}{|c|}{ Alto } & \multicolumn{2}{|c|}{ Total } & \multirow[t]{2}{*}{ Valor $\mathbf{p}$} \\
\hline & $\mathbf{n}$ & $\%$ & $\mathbf{n}$ & $\%$ & $\mathbf{n}$ & $\%$ & $\mathbf{n}$ & $\%$ & $\mathbf{n}$ & $\%$ & \\
\hline \multicolumn{12}{|c|}{ Grupo de edad } \\
\hline 19-34 años & 56 & 19,3 & 48 & 16,8 & 30 & 10,4 & 19 & 6,6 & 154 & 53,1 & \multirow{4}{*}{$0,0001^{*}$} \\
\hline 35-44 años & 17 & 5,9 & 19 & 6,6 & 20 & 6,9 & 11 & 3,8 & 67 & 23,1 & \\
\hline 45-64 años & 5 & 1,7 & 15 & 5,2 & 29 & 10,0 & 20 & 6,9 & 69 & 23,8 & \\
\hline Total & 78 & 26,9 & 82 & 28,5 & 79 & 27,3 & 50 & 17,2 & 290 & 100,0 & \\
\hline \multicolumn{12}{|l|}{ Sexo } \\
\hline Hombres & 34 & 11,7 & 37 & 12,8 & 30 & 10,3 & 27 & 9,3 & 128 & 44,1 & \multirow{3}{*}{0,515} \\
\hline Mujeres & 43 & 14,8 & 49 & 16,9 & 48 & 16,6 & 22 & 7,6 & 162 & 55,9 & \\
\hline Total & 77 & 26,5 & 86 & 29,7 & 78 & 26,9 & 49 & 16,9 & 290 & 100,0 & \\
\hline \multicolumn{12}{|l|}{ Residencia } \\
\hline Guadalajara & 53 & 18,2 & 53 & 18,2 & 50 & 17,3 & 33 & 11,4 & 189 & 65,1 & \multirow{7}{*}{0,904} \\
\hline Zapopan & 8 & 2,8 & 13 & 4,5 & 14 & 4,9 & 6 & 2,0 & 41 & 14,2 & \\
\hline Tlajomulco & 3 & 1,0 & 3 & 1,0 & 3 & 1,0 & 3 & 1,0 & 12 & 4,0 & \\
\hline Tlaquepaque & 3 & 1,0 & 4 & 1,4 & 4 & 1,4 & 3 & 1,0 & 14 & 4,8 & \\
\hline Tonalá & 3 & 1,0 & 4 & 1,4 & 3 & 1,0 & 3 & 1,0 & 13 & 4,4 & \\
\hline $\mathrm{RFE}^{1}$ & 7 & 2,4 & 7 & 2,4 & 5 & 1,7 & 3 & 1,0 & 22 & 7,5 & \\
\hline Total & 77 & 26,4 & 84 & 28,9 & 79 & 27,3 & 50 & 17,4 & 290 & 100,0 & \\
\hline \multicolumn{12}{|l|}{ Escolaridad } \\
\hline E. Básica & 5 & 1,7 & 7 & 2,5 & 8 & 2,8 & 4 & 1,3 & 24 & 8,3 & \multirow{5}{*}{0,917} \\
\hline Preparatoria & 12 & 4,1 & 15 & 5,1 & 11 & 3,7 & 5 & 1,7 & I & 14,6 & \\
\hline Licenciatura & 45 & 15,5 & 43 & 14,8 & 45 & 15,6 & 28 & 9,7 & 161 & 55,6 & \\
\hline Posgrado & 14 & 4,9 & 20 & 6,9 & 16 & 5,6 & 12 & 4,1 & 62 & 21,5 & \\
\hline Total & 76 & 26,2 & 85 & 29,3 & 80 & 27,7 & 49 & 16,8 & 290 & 100,0 & \\
\hline
\end{tabular}

Niveles OMS; Muy bajo <4.9; Bajo 5,0-8,9; Moderado 9,0-13,9; Alto >14,0. CPOD; Índice de dientes cariados, perdidos y obturados. Se realizó un análisis de Chi-cuadrado para mostrar la dependencia entre la categorización de los niveles de CPOD y las variables sociodemográficas. *Significancia estadística $p<0,05 .{ }^{1}$ RFE: Regiones Foráneas del Estado (Localidades fuera de la Zona Metropolitana de Guadalajara, que se encuentren dentro del estado de Jalisco, México). 
en el CPOD global entre los grupos de con hábito de fumar, hábito de alcohol, ambos y ninguno (Tabla 3).

\section{Discusión}

Se realizó el análisis en base a los niveles de severidad del índice CPOD de la OMS y las características sociodemográficas, es la edad la que muestra una diferencia significativa ( $p=0,0001$; Chi-2). En cuanto a los otros datos sociodemográficos como sexo $(p=0,585$; Chi$2)$, lugar de residencia $(p=0,906$; Chi-2,), escolaridad ( $p=0,930$; Chi-2) no hubo diferencia significativa. La discrepancia elevada se encontró en el grupo de mayor edad el cual se concentra en los niveles moderado y alto, mientras que los grupos de menor edad se concentran en su mayoría en muy bajo y bajo, es debido a la evolución natural de la enfermedad con el tiempo debido a su carácter infeccioso crónico. Mientras que en los datos sociodemográficos restantes la distribución grupal es relativamente homogénea.

De acuerdo con los datos del Sistema de Vigilancia Epidemiológica de Patologías bucales (SIVEPAB) ${ }^{14}$ en México, el índice CPOD de la población adulta, mayor a 50 años se encuentra entre 16,6-17,9, con una mayor experiencia de caries no tratadas, observándose en dicho sector poblacional niveles elevados de CPOD, ${ }^{(14)}$ lo cual no coincide con los datos del presente estudio $(10,1-12,7)$, en el cual la muestra de pobladores adultos mostró niveles bajos de caries, posiblemente por poseer un ingreso estable y seguro de salud. Se refuerza esto también al comparar con el estudio realizado por Olmos et al. ${ }^{15}$ en población adulta uruguaya, en el que encontraron un índice CPOD de 12,4 (IC 95\%: 11,912,9), distinto al de nuestra población de 8,3 (IC 95\%: 7,6-8,9).

En 2017, Heredia-Ponce et al. ${ }^{16}$ dirigieron un estudio en mujeres mexicanas adultas mayores institucionalizadas con diferentes niveles socioeconómicos, y aunque las diferencias entre dientes perdidos y obturados eran significativas entre los grupos, se encontraban elevados en ambos, independientemente de su grado socioeconómico ${ }^{15}$, al igual que en el presente estudio, se observaron niveles más altos de dientes perdidos y obturados debido la progresión de la secuela de enfermedad no tratada como se observó también en nuestro grupo de mayor edad ${ }^{17}$.

Por otro lado, es importante destacar que en nuestro estudio el componente de cariados del índice se mostró significativamente mayor en el grupo de fumadores en comparación con el de no fumadores, lo que coincide con autores que han relacionado el hábito tabáquico con el aumento de caries dental en la población adulta, y que en algunos casos se ha considerado que incluso la puede duplicar ${ }^{9,10}$.

En base a los resultados del presente estudio, las obturaciones se ven incrementadas tanto en el grupo de mayor edad, como en la población con escolaridad máxima de posgrado, lo que indica que la secuela de la enfermedad fue tratada a lo largo de las etapas de vida, y esto se dio en mayor proporción en los niveles educativos superiores.

Aun cuando arrojaron experiencia de caries todos los participantes, el CPOD fue bajo y el grupo de mayor edad se destacó por su CPOD moderado, lo cual es menor al observado en la población mexicana en general con características menos favorables, lo que se puede deber a que estos trabajadores cuentan con un salario fijo que les podría permitir un mayor acceso a servicios de salud, educación en salud y los propios servicios de salud proporcionados por la naturaleza de su labor en el ámbito académico.

Los resultados de este estudio plantean un panorama de comprensión sobre la experiencia de caries que afecta a un sector de población adulta en México que podría ser

Tabla 3. Índice CPOD de acuerdo con hábitos de tabaco y alcohol

\begin{tabular}{|c|c|c|c|c|c|c|c|c|c|}
\hline Tabaco & $\mathbf{n}$ & $\mathrm{C}$ & IC $95 \%$ & $\mathbf{P}$ & IC $95 \%$ & $\mathrm{O}$ & IC $95 \%$ & CPOD & IC $95 \%$ \\
\hline Sí & 57 & $5,2 \pm 0,6$ & $3,6-6,2$ & $1,2 \pm 0,2$ & $0,6-1,7$ & $3,3 \pm 0,7$ & $1,7-4,7$ & $9,7 \pm 0,9$ & $7,6-11,15$ \\
\hline No & 233 & $3,9 \pm 0,2$ & $3,4-4,3$ & $1,0 \pm 0,1$ & $0,7-1,2$ & $3,1 \pm 0,2$ & $2,6-3,6$ & $8,0 \pm 0,3$ & $7,3-8,7$ \\
\hline Valor de $p$ & & $0,034^{a^{*}}$ & & $0,616^{\mathrm{a}}$ & & $0,662^{\mathrm{a}}$ & & $0,057^{\mathrm{a}}$ & \\
\hline \multicolumn{10}{|l|}{ Alcohol } \\
\hline Sí & 132 & $4,3 \pm 0,3$ & $3,6-5,0$ & $0,9 \pm 0,1$ & $0,6-1,2$ & $3,0 \pm 0,4$ & $2,2-3,7$ & $8,2 \pm 0,5$ & $7,2-9,2$ \\
\hline No & 158 & $3,9 \pm 0,3$ & $3,2-4,4$ & $1,2 \pm 0,1$ & $0,8-1,5$ & $3,3 \pm 0,3$ & $2,6-3,9$ & $8,3 \pm 0,4$ & $7,5-9,2$ \\
\hline Valor de $p$ & & $0,421^{\mathrm{a}}$ & & $0,269^{a}$ & & $0,576^{a}$ & & $0,854^{\mathrm{a}}$ & \\
\hline \multicolumn{10}{|c|}{$\begin{array}{l}\text { Hábitos } \\
\text { Perniciosos }^{\dagger}\end{array}$} \\
\hline Alguno & 139 & $4,3 \pm 0,2$ & $3,6-5,0$ & $0,9 \pm 0,1$ & $0,6-1,2$ & $3,0 \pm 0,3$ & $2,3-3,8$ & $8,3 \pm 0,4$ & $7,3-9,2$ \\
\hline Ninguno & 151 & $3,8 \pm 0,3$ & $3,2-4,4$ & $1,1 \pm 0,1$ & $0,8-1,5$ & $3,2 \pm 0,3$ & $2,5-3,9$ & $8,2 \pm 0,4$ & $7,3-9,1$ \\
\hline Valor dep & & $0,860^{\mathrm{a}}$ & & $0,227^{a}$ & & $0,574^{a}$ & & $0,531^{\text {a }}$ & \\
\hline
\end{tabular}

C; Cariados, P; Perdidos, O; Obturados, CPOD; Índice de dientes cariados, perdidos y obturados; ${ }^{\mathrm{a}}$ t-Student (Intervalo de Confianza $95 \%)$ * *Significancia estadística $p=<0,05$. 'Se clasificaron en Alguno; si presentaban algún hábito o ambos y, Ninguno; si no presentaban ninguno de los dos. 
evaluado como no comprometido socialmente, pero a pesar de sus características sociodemográficas y acceso a los sistemas de salud la población estudiada se encontró distribuido de forma equilibrada en las distintas escalas de severidad del índice CPOD.

Aunque los datos que presentamos en este estudio podrían ser útiles para dirigir estrategias de salud, especialmente cuando los datos epidemiológicos en adultos y trabajadores mexicanos se encuentran tan escasos, se reconoce que el presente estudio fue hecho bajo las limitaciones de un bajo índice kappa inter-observador el cual se debió mejorar. También, nos encontramos con dificultades para la aceptación de la exploración oral, la cual fue de menos de la mitad de los participantes invitados, esto posiblemente derivado del lugar en donde se realizó la exploración, el cual era dentro de sus lugares de trabajo.

El índice CPOD poblacional se encontró en un nivel bajo, sin embargo, el grupo de mayor edad se mostró significativamente por encima de los dos grupos de menor edad. Se analizaron las diferencias entre los componentes por sexo, zona de residencia y escolaridad, encontrando los niveles del componente cariados significativamente más bajos en los habitantes de las regiones foráneas del estado y los que tenían escolaridad de posgrado.

\section{Referencias bibliográficas}

1. Wong A, Subar PE, Young DA. Dental Caries: An Update on Dental Trends and Therapy. Adv Pediatr. 2017;64(1):307-30. DOI: 10.1016/j.yapd.2017.03.011.

2. Chaffee BW, Rodrigues PH, Kramer PF, Vítolo MR, Feldens CA. Oral health-related quality-of-life scores differ by socioeconomic status and caries experience. Community Dent Oral Epidemiol. 2017;45(3):216-24. DOI: 10.1111/cdoe.12279.

3. Schwendicke F, Dörfer CE, Schlattmann P, Page LF, Thomson WM, Paris S. Socioeconomic Inequality and Caries: A Systematic Review and Meta-Analysis. J Dent Res. 2014;94(1):10-8. DOI: 10.1177/0022034514557546.

4. Molina-Frechero N, Durán-Merino D, Castañeda-Castaneira E, Juárez-López ML. Dental caries experience and its relation to oral hygiene in Mexican children. Gac Med Mex. 2015;151(4):485-90.

5. Frencken JE, Sharma P, Stenhouse L, Green D, Laverty D, Dietrich T. Global epidemiology of dental caries and severe periodontitis - a comprehensive review. J Clin Periodontol. 2017;44(S18):S94-S105. DOI: 10.1111/ jcpe. 12677.

6. Seerig LM, Nascimento GG, Peres MA, Horta BL, Demarco FF. Tooth loss in adults and income: Systematic review and meta-analysis. J Dent. 2015;43(9):1051-9. DOI: $10.1016 /$ j.jdent.2015.07.004.
7. Vano M, Gennai S, Karapetsa D, Miceli M, Giuca MR, Gabriele M, et al. The influence of educational level and oral hygiene behaviours on DMFT index and CPITN index in an adult Italian population: an epidemiological study. Int J Dent Hyg. 2015;13(2):151-7. DOI: 10.1111/idh.12098.

8. Rooban T, Vidya K, Joshua E, Rao A, Ranganathan S, Rao UK, et al. Tooth decay in alcohol and tobacco abusers. J Oral and Maxillofac Pathol. 2011;15(1):14-21. DOI: $10.4103 / 0973-029 X .80032$.

9. De-la-Cruz-Cardoso D, Castillo Rojano G, Cervantes Sandoval A. Prevalencia de caries dental asociada a tabaquismo en una población de universitarios. Rev ADM. 2017;74(1):11-6.

10. Torres-Remírez J, Ibáñez M. La Relación entre la Salud Oral y el Consumo de Tabaco. Un Estudio de los Habitantes de la Rioja Alta. Int J Estomatol. 2020;14:95-100. DOI: $10.4067 /$ S0718-381X2020000100095.

11. Asociación Médica Mundial. Declaración de Helsinki de la AMM-Principios éticos para las investigaciones médicas en seres humanos. 2019. [Consultado el 08 de marzo del 2019]. Disponible en: https://www.wma.net/es/ policies-post/declaracion-de-helsinki-de-la-amm-principios-eticos-para-las-investigaciones-medicas-en-seres-humanos/.

12. World Health Organization. Oral health surveys: Basic methods. 5th ed. Brazil: University of São Paulo; 2013. 73-76.

13. Krippendorff K. Content analysis: An introduction to its methodology: Sage publications; 2018.Chapter 11. Reliability. 211-256.

14. Mejía-González AM, Rodríguez-González KG, Lomelí-Buyoli G. Resultados del Sistema de Vigilancia Epidemiológica de Patologías Bucales Sivepab 2015. Ciudad de México: Secretaría de Salud, Dirección General de Epidemiología; 2015.70 p.

15. Olmos P, Piovesan S, Musto M, Lorenzo S, Álvarez R, Massa F. Caries dental. La enfermedad oral más prevalente Primer Estudio poblacional en jóvenes y adultos uruguayos del interior del país. Odontoestomatol. 2013;15(especial):26-34

16. Heredia-Ponce E, Irigoyen-Camacho AE, Sánchez-García S. Oral Health Status of Institutionalized Older Women from Different Socioeconomic Positions. J Health Care Poor Underserved. 2017;28(4):1462-76. DOI: 10.1353/hpu.2017.0127.

17. Quinteros ME, Cáceres DD, Soto A, Marino RJ, Giacaman RA. Caries experience and use of dental services in rural and urban adults and older adults from central Chile. Int Dent J. 2014;64(5):260-8. DOI: 10.1111/ idj. 12118 . 